\section{Childhood weight gain and oral microbiota}

The link between obesity and the oral and gut microbiota is well established in adults and adolescents, but is not well studied in children. A new paper published in Scientific Reports aimed to redress this imbalance, and found that the composition of the oral microbiota is associated with weight trajectories over the first 2 years of life.

"Together with our co-author paediatrician Ian Paul, we have been following a cohort of almost 400 children from their birth to the age of 3 years as part of the INSIGHT study," explains author Kateryna Makova. Some parts of the INSIGHT study have focused on the behavioural and dietary variables that influence the development of obesity, while the present paper focused on characterizing the oral and gut microbiota.

The researchers analysed oral and stool samples from $\sim 200$ children and measured weight and length at seven time points. "We used functional data analysis to analyse childhood weight-gain trajectories longitudinally," says author Matthew Reimherr. In children who gained weight rapidly, bacterial diversity was decreased and the Firmicutes to
Bacteroidetes ratio was increased in the oral microbiota, but not the gut microbiota. This pattern is known to occur in the gut microbiota of adults with obesity, so the finding suggests that an obesity-associated microbiota composition is established in the oral microbiota before it becomes established in the gut microbiota. "The links we were able to demonstrate between oral microbiota and weight gain are novel, and could lead to non-invasive clinical screens to identify children at a particular risk of obesity, who would be candidates for early preventive interventions," says author Francesca Chiaromonte.

The team are now planning to expand their study, including analyses of the siblings and mothers of the original cohort. "Expanding the study will allow us to have a fuller understanding of early life microbiota development and how that influences child growth," concludes author Sarah Craig.

\section{Claire Greenhill}

ORIGINAL ARTICLE Craig, S.J. et al. Child weight gain trajectories linked to oral microbiota composition. Sci. Rep. 8 , $14030(2018)$

\title{
NUTRITION
}

\section{Metabolic adaptations to high-fat diet}

"Dietary fat, particularly saturated fat, has been considered harmful for decades," explains senior author Bente Kiens. "However, more and more epidemiological studies refute this 'fat scare', but no solid mechanistic intervention studies in humans have been conducted to underpin such statements." Now, new research shows that eating a high-fat diet (HFD) that is calorically balanced causes metabolic adaptations that prevent metabolic complications, such as reduced insulin sensitivity.

The authors conducted a randomized parallel intervention study and assigned 18 healthy, untrained and slightly overweight men to either a HFD rich in polyunsaturated or saturated fat for 6 weeks. Both diets were eucaloric to maintain bodyweight.

Kiens and colleagues observed that participants in both diet intervention groups maintained their insulin sensitivity at the whole-body level. Analysis of skeletal muscle biopsy samples taken from the volunteers showed that the expression of lipid transporters and intracellular fatty acid handling proteins were increased after the HFD intervention. Fatty acid oxidation was also elevated, suggesting that in response to a HFD skeletal muscles induced fatty acid uptake and oxidation instead of fat storage. Insulin sensitivity in the skeletal muscle was unaffected by HFD, which supported the observation that insulin sensitivity was maintained at the whole-body level. Additionally, hepatic insulin clearance increased and gluconeogenesis and fasting plasma levels of triacylglycerol decreased, suggesting a change towards a non-diabetic direction.

Furthermore, mice fed the same HFD as humans also maintained their insulin sensitivity. Analysis of liver-specific changes in mice fed a HFD suggests that the liver decreases gluconeogenesis and lipogenesis to adapt to a diet rich in fat. "Thus, if you avoid caloric surplus then a fat-rich diet, be it polyunsaturated or saturated, is not detrimental to health, but in fact has several health benefits." concludes Kiens.

Ivone Leong

ORIGINAL ARTICLE Lundsgaard, A.-M. et al. Mechanisms preserving insulin action during high dietary fat intake. Cell Metab. https://doi.org/10.1016/j.cmet.2018.08.022 (2018)

\section{Effects of PTH on bone composition}

The lacunar-canaliculi system is an extensive network in the mineralized matrix of the bone and is created and maintained by osteocytes, which reside in the lacunar spaces.

Osteocytes can change the local tissue composition and tissue strength by modifying the lacunar spaces. Chronic exposure to parathyroid hormone (PTH) causes changes at the lacunar wall; however, little is known about the effect of intermittent PTH treatment on the perilacunar. Now, new research by David Kohn and co-workers shows that intermittent PTH exposure causes substantial tissue composition changes that result in increased tissue strength and resistance to microdamage (small fractures).

To investigate the effect of intermittent PTH treatment on perilacunar adaptation and changes in tissue composition, the researchers treated mice with either PTH(1-34) or vehicle control for 3 weeks. After treatment, the mice were assessed using Raman spectroscopy. The team found substantial changes to the perilacunar composition, including decreased mineral to matrix ratio at the lacuna wall and decreases in carbonate to phosphate ratio and crystallinity across the bone tissue in mice treated with $\mathrm{PTH}(1-34)$ compared with controls.

Next, the team analysed the contralateral limb to understand the consequences of the changes to the perilacunar on structural and tissue mechanical properties. At the structural level, the stiffness of the tibiae increased in mice treated with $\mathrm{PTH}(1-34)$ despite no changes to bone mass and cross-sectional geometry. This increase corresponded with increased modulus and tissue strength.

Finally, Kohn and colleagues investigated whether the changes induced by PTH(1-34) increased resistance to microdamage. During fatigue loading (a process where the tested material is continuously loaded with a force until cracking occurs), both test groups had substantial loss in stiffness. However, after fatigue loading mice treated with PTH(1-34) had less microdamage than control mice. Taken together, these results suggest that intermittent treatment with PTH(1-34) induces changes at the lacunar wall, which increases bone strength and its resistance to microdamage.

Ivone Leong

ORIGINAL ARTICLE Gardinier, J. D. et al. Examining the influence of PTH(1-34) on tissue strength and composition. Bone 117, 130-137 (2018) 